\title{
Impact of the 'Giving Cigarettes is Giving Harm' campaign on knowledge and attitudes of Chinese smokers
}

\author{
Li-Ling Huang, ${ }^{1}$ James F Thrasher, ${ }^{1}, 2$ Yuan Jiang, ${ }^{3}$ Qiang Li, ${ }^{3,4}$ Geoffrey T Fong, ${ }^{3,4,7}$ \\ Yvette Chang, ${ }^{6}$ Katrina M Walsemann, ${ }^{1}$ Daniela B Friedman ${ }^{1}$
}

${ }^{1}$ Department of Health Promotion, Education, and Behavior, Arnold School of Public Health, University of South Carolina, Columbia, South Carolina, USA ${ }^{2}$ Center for Population Health Research, National Institute of Public Health, Cuernavaca, Mexico

${ }^{3}$ National Tobacco Control Office, Chinese Center for Disease Control and Prevention, Beiijing, China ${ }^{4}$ Department of Psychology, University of Waterloo, Waterloo, Ontario, Canada ${ }^{5}$ Ontario Institute for Cancer Research, Toronto, Ontario, Canada

${ }^{6}$ World Lung Foundation, New York City, New York, USA ${ }^{7}$ School of Public Health and Health Systems, University of Waterloo, Waterloo, Ontario, Canada

Correspondence to Li-Ling Huang.

Department of Health Promotion, Education, and Behavior, Arnold School of Public Health, University of South Carolina, 915 Greene Street, Room 524, Columbia, SC 29208, USA:

huang|@mailbox.sc.edu

Received 5 December 2013 Accepted 14 April 2014

Published Online First 9 May 2014

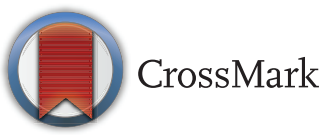

To cite: Huang $L-L$, Thrasher JF, Jiang $Y$, et al. Tob Control 2015;24: iv28-iv34.

\section{ABSTRACT}

Objective To date there is limited published evidence on the efficacy of tobacco control mass media campaigns in China. This study aimed to evaluate the impact of a mass media campaign 'Giving Cigarettes is Giving Harm' (GCGH) on Chinese smokers' knowledge of smokingrelated harms and attitudes towards cigarette gifts. Methods Population-based, representative data were analysed from a longitudinal cohort of 3709 adult smokers who participated in the International Tobacco Control (ITC) China Survey conducted in six Chinese cities before and after the campaign. Logistic regression models were estimated to examine associations between campaign exposure and attitudes towards cigarette gifts measured post-campaign. Poisson regression models were estimated to assess the effects of campaign exposure on post-campaign knowledge, adjusting for pre-campaign knowledge.

Findings Fourteen percent $(n=335)$ of participants recalled the campaign within the cities where the GCGH campaign was implemented. Participants in the intervention cities who recalled the campaign were more likely to disagree that cigarettes are good gifts ( $71 \%$ vs $58 \%, p<0.01)$ and had greater levels of campaigntargeted knowledge than those who did not recall the campaign (mean $=1.97$ vs $1.62, p<0.01$ ). Disagreeing that cigarettes are good gifts was higher in intervention cities than in control cities. Changes in campaigntargeted knowledge were similar in both cities, perhaps due to a secular trend, low campaign recall or contamination issues.

Conclusions These findings suggest that the GCGH campaign increased knowledge of smoking harms, which could promote downstream cessation. This study provides evidence to support future campaign development to effectively fight the tobacco epidemic in China.

\section{INTRODUCTION}

The WHO's Framework Convention on Tobacco Control recommends implementing national antismoking mass media campaigns to educate the public about the dangers of tobacco use. ${ }^{1}$ Mass media campaigns significantly reduce smoking initiation among youth, increase smoking cessation among adults, decrease the social acceptability of smoking and establish smoke-free norms. ${ }^{2-9}$ Most studies of mass media campaigns have been conducted in high-income countries and have shown consistently that anti-smoking advertisements that arouse strong emotions, display graphic, serious consequences from smoking, and/or use highly emotional testimonials have greater impact than those without such features. ${ }^{2} 36$ 10-12 Similarly, emerging evidence from population-based studies suggests that graphic portrayals of serious smoking consequences are effective with smokers in India and Russia. ${ }^{13}{ }^{14}$ Formative research on antismoking advertisement strategies in ten low-income and middle-income countries (LMICs), including China, found that emotionally arousing graphic messages (ie, strong graphic and visceral imagery) to depict serious consequences of smoking are most likely to be perceived as effective. ${ }^{9}$ Although Chinese smokers perceived the graphic ads as effective, they were less likely to discuss the ads than smokers from other countries. ${ }^{9}$ The variable responses to certain types of messaging strategies across countries highlight the critical need for evaluation of tobacco control mass media campaigns to ensure cultural appropriateness and maximise their effectiveness. Population-based research in LMICs like China is needed to assess whether strongly emotional ads with graphic serious consequences from smoking are effective when adapted to specific sociocultural contexts.

China is the largest cigarette market in the world, with about 301 million smokers who represent a third of the world's smokers and who consume $38 \%$ of the world's cigarettes. ${ }^{15}{ }^{16}$ According to data from the 2010 Global Adult Tobacco Survey in China, most Chinese men smoke (52.9\%), whereas very few Chinese women smoke $(2.4 \%) .{ }^{15}$ The Chinese government has not made tobacco control a high priority in its health reform plan and has allocated only $0.5 \%$ of its disease control and prevention budget to tobacco control efforts, even though it has ratified the WHO's Framework Convention on Tobacco Control. ${ }^{17}$ Indeed, the State Tobacco Monopoly Administration is the authority in China that regulates health warning labels on cigarette packaging and oversees China National Tobacco Corporation. ${ }^{17}$ As a state-owned monopoly and the world's largest and most profitable tobacco company, ${ }^{18}$ the Chinese government has conflicting interests around the regulation of tobacco production/marketing and tobacco control. ${ }^{17}$ 19-21

Few large-scale anti-smoking mass media campaigns were implemented in China before 2008. In 2008, the Chinese government launched subnational anti-smoking mass media campaigns, that is, 'Smoke-free Beijing' (SFB) and 'Smoke-free Olympics' (SFO), to discourage smoking, particularly in smoke-free places, in order to fulfil its obligation of ensuring a smoke-free Beijing Olympics. SFO campaign materials usually involved positive, 
celebratory tones, used humorous appeals, and conveyed limited information about smoking-related harms. Some SFB campaign materials were similar, but other materials featured graphic depiction of smoking harms. Scarce published and anecdotal evidence suggests that some campaigns were relatively successful, ${ }^{22}$ although one study suggests that the effects of SFO campaigns were limited and did not significantly reduce smoking in workplaces and restaurants. ${ }^{23}$ This lack of reduction in smoking in key public venues over time (2006-2009) was also found by the China administration of the International Tobacco Control (ITC) China Survey. ${ }^{24}$

In partnership with the WHO and World Lung Foundation, China launched the 'Giving Cigarettes is Giving Harm' (GCGH) campaign in 2009 to raise awareness of tobacco-attributed diseases and reduce the social acceptability of giving cigarettes as gifts, a common practice for establishing and maintaining interpersonal relationships in Chinese society. ${ }^{25} 26$ Gifting and sharing cigarettes significantly promotes smoking and hinders cessation efforts among Chinese smokers. ${ }^{25} 27$ To discourage people from gifting cigarettes, campaign messages equated gifting cigarettes to loved ones and colleagues with giving them omens that portend future diseases and death from smoking. The campaign's novel strategy of situating graphic imagery of harm within the context of a socially engrained and respected practice warrants evaluation. Limited evidence suggests that the campaign was effective in raising the awareness of smoking-attributed diseases among the Chinese. ${ }^{28}$ The evaluation studies of tobacco control media campaigns in China were limited by design issues such as non-representative samples, the lack of control groups, and the inability to determine associations between campaign exposure and individual-level change in campaign-targeted outcomes due to using repeated cross-sectional designs. Formative pretesting of messages in 10 LMICs including China provided preliminary evidence of message types that are likely to be effective in China-namely, those that use strong graphic and visceral imagery to depict serious consequences of smoking. ${ }^{9}$ However, this formative study did not evaluate messaging strategies under naturalistic conditions of exposure.

The present study aims to overcome limitations of prior research by using a population-based, longitudinal cohort of adult smokers to evaluate one of China's first anti-smoking mass media campaign to graphically portray tobacco-attributed diseases and to attempt to change social norms around cigarette gifting. We hypothesised that campaign exposure would be positively associated with increases in campaign-targeted knowledge and negative attitudes towards cigarettes as gifts. The results will strengthen emerging evidence regarding effective graphic and culturally adapted campaign content in China, which can be used to develop future campaigns to fight the tobacco epidemic in China.

\section{METHODS}

\section{Study design}

This study used a quasi-experimental design with a populationbased, longitudinal cohort of 3709 adult smokers who participated in the ITC China Survey, which was conducted in six Chinese cities before and after the campaign. We assessed campaign-targeted knowledge and attitudes using two different analytical samples: (1) comparing cities where the GCGH campaign was implemented (Beijing, Shenyang, Shanghai, Guangzhou) with cities where it was not implemented (Yinchuan, Changsha); (2) within the intervention cities, comparing those who reported exposure with those who did not. Furthermore, we examined associations between campaigntargeted knowledge and attitudes and the number and type of media channels (television (TV), posters, mobile media) through which participants recalled campaign exposure within the intervention cities. Yinchuan and Changsha were coded as control cities, because the campaign (including posters) was not specifically implemented there; however, there may have been some contamination through satellite TV.

\section{Study sample}

A stratified multistage cluster sampling design was used to select a population-based, representative sample of approximately 800 adult smokers in each of six Chinese cities that were included in the ITC China Survey, designed to be parallel to surveys being conducted in 21 other countries of the ITC Project. ${ }^{29}$ To the extent possible, participants were followed and interviewed again, but in order to maintain sample size over time, participants lost to follow-up were replaced using the same sampling frame constructed at Wave $1 .^{29}$ For the current study, we analysed data from Wave 2, which were collected from October 2007 to January 2008, one year before the campaign started, and from Wave 3, collected from May to September 2009, a period that started 3 months after the campaign ended. Adult smokers were defined as those who were 18 years or older, had smoked at least 100 cigarettes in their lifetime, and were currently smoking at least once a week. A total of 4732 smokers were surveyed at the Wave 1 with response rates from $39 \%$ to $61 \%$ and cooperation rates from $80 \%$ to $95 \%$ in six cities. ${ }^{29}$ The average retention rates for Wave 2 and Wave 3 were $81.6 \%$ and $80.4 \%$. The 3709 smokers who completed Wave 2 and Wave 3 surveys constituted the analytical sample for this study. Hereafter these two waves are referred to as 'baseline' or 'pre-campaign' and 'follow-up' or 'post-campaign.' Additional information can be found in the ITC China Survey Technical Report. ${ }^{30}$

\section{Campaign materials and channels}

The GCGH campaign included a 30-s TV advertisement and three posters. ${ }^{31} 32$ The campaign aired on regional and satellite TV, mobile media on city buses and subway trains, on outdoor electronic billboards, and in hospitals and community centres for four weeks from January through February 2009 in Beijing, Shanghai, Tianjin, Shenyang, Guangzhou, Shenzhen and Shaoguan. ${ }^{33}$ During the same period, the posters were also distributed to more than 30 cities where media broadcast was not achieved. ${ }^{33}$ The TV advertisement and one poster used the same message, which equated lung cancer, respiratory diseases, heart disease, stroke and cardiovascular diseases with cigarette products. ${ }^{\text {ii }}$

\section{Measurements}

\section{Campaign-targeted knowledge and attitudes}

Knowledge of smoking-related harms was assessed in the precampaign and post-campaign surveys. Participants indicated whether they believed that smoking causes (1) lung cancer in smokers, (2) strokes and (3) cardiovascular disease. Participants indicated yes or no to each item, and an index was created for campaign-targeted knowledge with values ranging from 0 to 3 .

Attitude towards cigarettes as gifts was assessed by the postcampaign survey only. Participants were asked how much they

${ }^{\mathrm{i}}$ Mobile media refers to television screens where advertisements are placed on city buses and subway trains.

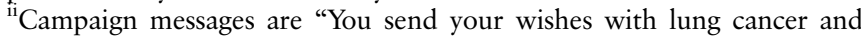
other respiratory diseases to your friends; you send your respects with heart disease, stroke, and other cardiovascular diseases to your colleagues; you send your caring with death to your family members." 33 
agreed that cigarettes are good gifts for friends and family on a 5 -point Likert scale, and the responses were dichotomised to reflect agreement or not (strongly disagree and disagree vs agree, strongly agree, and neither disagree nor agree).

\section{Campaign exposure measures}

Exposure to the GCGH campaign was assessed with an aided recall question at the post-campaign survey only: "Have you ever seen the campaign 'Giving Cigarettes is Giving Harm'?" Participants were asked about their past exposure to the GCGH campaign, without any other visual presentation and verbal description of the campaign content. For those who recalled the campaign, exposure through each of three media was assessed: TV, poster and mobile media, and dummy variables were created for each of these channels with no recall of exposure to the campaign coded as 0 . A three-level campaign exposure index was created to indicate no exposure to the campaign (the reference group), exposure to one channel, or exposure to two or more channels.

\section{Adjustment variables}

Sociodemographic variables were assessed pre-campaign and included age, sex, marital status, monthly household income (low $=¥ 3000$ or less; medium $=¥ 3001-5000$; high $=¥ 5001$ or more), and education (low=elementary school or less; medium=junior high school and high school; high=college/university or more). Smokers were categorised into daily and non-daily smokers. The heaviness of smoking index was calculated using information on daily cigarette consumption as well as the time elapsed from waking to smoking the first cigarette of the day, with scores ranging from 0 to $6 .{ }^{34}$ Intention to quit smoking was measured by whether participants planned to quit within the next six months or not. Exposure to general antismoking campaigns was assessed by whether participants reported any exposure to such campaigns in the six months prior to the interview in the post-campaign survey. Survey time was assessed in days from the campaign end until the time of the post-campaign survey for each individual.

\section{Analysis}

The analyses were conducted using STATA, V.11.2. ${ }^{35}$ The attrition analysis involved using unadjusted data and conducting $\chi^{2}$ tests and $t$ tests to examine differences among participants who were followed up and those who were lost to attrition between the two waves. All other analyses accounted for the multistage, cluster sampling design and for sampling weights developed for the longitudinal sample. ${ }^{29}$ Logistic regression was used to examine associations between campaign exposure and attitudes. Linear regression was conducted as a sensitivity analysis to include full range of response options for the attitudes outcome. Poisson regression was used to examine associations between campaign exposure and post-campaign levels of knowledge, adjusting for pre-campaign levels of knowledge. Since the variance of knowledge measures is slightly smaller than the mean $(1.1<1.7)$, robust SEs were obtained to control for minor violation of assumptions for Poisson distribution. Both types of models assessed crude and adjusted estimates of the relationship between exposure and outcomes. Adjusted estimates account for age, sex, income, education, marital status, smoking status, intention to quit, exposure to general anti-smoking campaign, and survey time from the campaign end until the time of the post-campaign survey.

\section{RESULTS}

\section{Sample characteristics}

The baseline characteristics of participants by follow-up status, residence in intervention or control cities, and campaign recall within the intervention cities are shown in Table 1. Those who were lost to follow-up $(\mathrm{n}=949)$ were more likely than those who were followed $(\mathrm{n}=3709)$ to be younger, have higher educational attainment and have lower household income. Participants in the intervention cities appeared older, had less variance in education and had lower levels of income compared with those who in the control cities. Those who did and did not recall the campaign within the intervention cities only differed in quit intention in the next six months and exposure to any anti-smoking campaign in the last six months.

\section{Campaign exposure assessment}

The prevalence of GCGH campaign recall was $14 \%$ in the intervention cities $(n=335)$. Among those who recalled the campaign $70 \%$ reported that they had seen the campaign on TV, $23 \%$ on mobile media and $25 \%$ on posters. When further analysing the number of the channels to which the 335 participants in the intervention cities reported that they were exposed, $61 \%$ of them recalled seeing the campaign on one channel and $24 \%$ recalled seeing the campaign on two or more channels. TV had more penetration than other channels since $76 \%$ of those who recalled seeing the campaign on one channel saw it on TV.

\section{Effectiveness of the GCGH campaign}

Campaign-targeted attitude towards cigarette gifts

The percentage of people who disagreed that cigarettes were good gifts was higher in the intervention compared with the control cities $(60 \%$ vs $55 \%, p=0.26)$, with a statistically significant difference in the adjusted model (adjusted OR (AOR) $=1.59,95 \% \mathrm{CI}$ 1.11 to 2.27; Table 2). Within the intervention cities, participants who recalled the campaign were more likely than those who did not recall the campaign to disagree that cigarettes were good gifts ( $71 \%$ vs $58 \%, \mathrm{p}<0.01 ; \mathrm{OR}=1.75,95 \%$ CI 1.28 to 2.41 ; $\mathrm{AOR}=1.63,95 \% \mathrm{CI} 1.18$ to 2.25$)$. Those who reported having seen the campaign advertisement on one channel were also more likely to disagree that cigarettes were good gifts $(\mathrm{OR}=2.09,95 \%$ CI 1.25 to 3.50 ; $\mathrm{AOR}=2.00,95 \%$ CI 1.22 to 3.29 ) compared with those who did not recall the campaign; but the significance was not shown among those who reported campaign exposure through two or more channels. Among the three channels through which participants recalled campaign exposure, only TV was significantly associated with attitude towards cigarettes as gifts in an unadjusted model (OR=1.62, 95\% CI 1.02 to 2.55). After adjusting for potential confounders, no exposure through any of the three channels was significantly associated with attitudes. The sensitivity analysis produced a consistent pattern of results.

\section{Campaign-targeted knowledge of smoking harms}

When comparing intervention and control cities, there was no statistically significant difference in campaign-targeted knowledge after adjusting for baseline levels of knowledge (Table 3). However, within intervention cities, we found campaign recall was statistically significantly and positively associated with campaigntargeted knowledge of smoking harms (unadjusted $\mathrm{b}=0.168$, $\mathrm{SE}=0.043, \mathrm{p}<0.001$; adjusted $\mathrm{b}=0.147, \mathrm{SE}=0.043, \mathrm{p}=0.001$ ).

The number of channels through which campaign exposure was reported was associated with relatively greater levels of campaign-targeted knowledge when compared with participants who did not recall the campaign in intervention cities. The 
Table 1 Sample sociodemographics and smoking characteristics by comparison groups

\begin{tabular}{|c|c|c|c|c|c|c|c|}
\hline Baseline characteristics & & $\begin{array}{l}\text { Followed } \\
n=3709\end{array}$ & $\begin{array}{l}\text { Not followed } \\
n=949\end{array}$ & $\begin{array}{l}\text { Intervention city } \\
\mathrm{n}=2585\end{array}$ & $\begin{array}{l}\text { Control city } \\
n=1124\end{array}$ & $\begin{array}{l}\text { Recalled in } \\
\text { intervention city } \\
n=335\end{array}$ & $\begin{array}{l}\text { Not recalled in } \\
\text { intervention city } \\
n=2239\end{array}$ \\
\hline $\operatorname{Age}^{*} \dagger$ & Average & 51.5 & 50.4 & 52.8 & 48.4 & 51.6 & 53.0 \\
\hline Sex $(\%)$ & Male & 94.7 & 95.8 & 94.7 & 94.6 & 94.3 & 94.7 \\
\hline Education $(\%)^{*} \dagger$ & $\begin{array}{l}\text { Low } \\
\text { Medium } \\
\text { High }\end{array}$ & $\begin{array}{l}12.2 \\
67.3 \\
20.5\end{array}$ & $\begin{array}{l}11.5 \\
63.0 \\
25.5\end{array}$ & $\begin{array}{l}11.3 \\
68.9 \\
19.8\end{array}$ & $\begin{array}{l}14.1 \\
63.8 \\
22.1\end{array}$ & $\begin{array}{r}9.9 \\
68.3 \\
21.8\end{array}$ & $\begin{array}{l}11.5 \\
68.9 \\
19.6\end{array}$ \\
\hline Monthly household income $(\%)^{*} \dagger$ & $\begin{array}{l}\text { Low } \\
\text { Medium } \\
\text { High }\end{array}$ & $\begin{array}{l}16.0 \\
48.8 \\
35.2\end{array}$ & $\begin{array}{l}19.4 \\
49.4 \\
31.2\end{array}$ & $\begin{array}{l}14.0 \\
47.2 \\
38.8\end{array}$ & $\begin{array}{l}20.6 \\
52.4 \\
27.0\end{array}$ & $\begin{array}{l}13.2 \\
45.1 \\
41.7\end{array}$ & $\begin{array}{l}14.2 \\
47.3 \\
38.5\end{array}$ \\
\hline Smoking status (\%) & $\begin{array}{l}\text { Daily } \\
\text { Non-daily }\end{array}$ & $\begin{array}{r}94.2 \\
5.8\end{array}$ & $\begin{array}{r}93.9 \\
6.1\end{array}$ & $\begin{array}{r}94.2 \\
5.8\end{array}$ & $\begin{array}{r}94.3 \\
5.7\end{array}$ & $\begin{array}{r}95.0 \\
5.0\end{array}$ & $\begin{array}{r}94.1 \\
5.9\end{array}$ \\
\hline HSI & Average & 2.33 & 2.34 & 2.35 & 2.27 & 2.33 & 2.35 \\
\hline Quit intention in the next 6 months (\%)‡ & Yes & 15.5 & 17.2 & 15.6 & 16.4 & 22.0 & 14.0 \\
\hline $\begin{array}{l}\text { Exposure to any anti-smoking campaign in the } \\
\text { last } 6 \text { months post-campaign (\%) } \ddagger\end{array}$ & Yes & 68.2 & - & 68.8 & 67.9 & 83.5 & 65.6 \\
\hline
\end{tabular}

strongest association was found for participants who recalled exposure to the campaign through two or more channels. This finding held in unadjusted $(b=0.301, \mathrm{SE}=0.069, \mathrm{p}<0.001)$ and adjusted models $(b=0.259 \mathrm{SE}=0.066, \mathrm{p}<0.001)$, with weaker but statistically significant associations found for those who recalled exposure through only one channel (Table 3).Campaign recall through TV was significantly and positively associated with post-campaign knowledge (unadjusted $b=0.131$, $\mathrm{SE}=0.044, \mathrm{p}=0.006$; adjusted $\mathrm{b}=0.106, \mathrm{SE}=0.040, \mathrm{p}=0.012$ ). Recall of the campaign through posters was positively associated with post-campaign knowledge only in the unadjusted model $(b=0.152, S E=0.074, p=0.047)$.

\section{DISCUSSION}

This study is the first large-scale population-based evaluation of a tobacco control mass media campaign conducted in China. The study findings suggest that recall of the GCGH campaign was associated with greater disapproval of gifting cigarettes and with greater increases in knowledge of smoking-related harms at the individual level among those who recalled the campaign within intervention cities. The campaign's novel strategy of linking cigarette gifting to images of diseased organs and symbols of death may have the potential to reduce the social acceptability of giving cigarettes as gifts. This result is consistent with previous studies which suggest that messages with graphic portrayals of smoking-related diseases are effective with smokers $23610-12$ and can be adapted to different sociocultural contexts. ${ }^{9}$ However, there was no difference between intervention and control cities in changes to knowledge after controlling for pre-campaign levels of knowledge. The individual-level effects would have had to be quite large to be detected at the population level. Possible explanations for non-existent effect at the population level could be the relatively low levels of campaign exposure, and potential contamination in the control cities where some participants may have

Table 2 Association between campaign exposure and campaign-targeted attitudes towards cigarette gifts

\begin{tabular}{|c|c|c|c|c|c|}
\hline \multirow[b]{2}{*}{ Attitude measure } & \multirow[b]{2}{*}{ Campaign exposure } & \multirow[b]{2}{*}{$\mathbf{n}$} & \multirow[b]{2}{*}{ Per cent } & \multicolumn{2}{|c|}{ Logistic regression ORs $(95 \% \mathrm{CI})$} \\
\hline & & & & Unadjusted & Adjusted \\
\hline \multirow{13}{*}{$\begin{array}{l}\text { Disagreeing that cigarettes are good gifts } \\
\text { for friends and family }\end{array}$} & Model 1 & & & & \\
\hline & Control cities & 965 & 55 & 1 & 1 \\
\hline & Intervention cities & 2333 & 60 & 1.22 (0.86 to 1.73$)$ & $1.59(1.11 \text { to } 2.27)^{*}$ \\
\hline & Model 2 & & & & \\
\hline & Not recalled & 2043 & 58 & 1 & 1 \\
\hline & $\begin{array}{l}\text { Recalled the campaign } \\
\text { Model } 3\end{array}$ & 282 & 71 & $1.75(1.28 \text { to } 2.41)^{\star *}$ & $1.63(1.18 \text { to } 2.25)^{* *}$ \\
\hline & Not recalled & 2043 & 58 & 1 & 1 \\
\hline & Recalledt 1 channel & 173 & 75 & $2.09(1.25 \text { to } 3.50)^{* *}$ & $2.00(1.22 \text { to } 3.29)^{* *}$ \\
\hline & $\begin{array}{l}\text { Recalled } 2 \text { and more channels } \\
\text { Model } 4\end{array}$ & 58 & 60 & $1.05(0.43$ to 2.55$)$ & $0.84(0.34$ to 2.09$)$ \\
\hline & Not recalled $\ddagger$ & 2043 & 58 & 1 & 1 \\
\hline & Recalled TV & 190 & 70 & $1.62(1.02 \text { to } 2.55)^{*}$ & $1.51(0.96$ to 2.37$)$ \\
\hline & Recalled poster & 54 & 62 & 0.84 (0.49 to 1.42$)$ & $0.80(0.44$ to 1.47$)$ \\
\hline & Recalled mobile media & 67 & 71 & 1.32 (0.67 to 2.60$)$ & 1.19 (0.62 to 2.26$)$ \\
\hline
\end{tabular}


Table 3 Association between campaign exposure and campaign-targeted knowledge of smoking harms

\begin{tabular}{|c|c|c|c|c|c|}
\hline \multirow[b]{2}{*}{ Knowledge measure } & \multirow[b]{2}{*}{ Campaign exposure } & \multirow{2}{*}{$\begin{array}{l}\text { Pre- } \\
\text { campaign } \\
\text { mean }\end{array}$} & \multirow{2}{*}{$\begin{array}{l}\text { Post- } \\
\text { campaign } \\
\text { mean }\end{array}$} & \multicolumn{2}{|c|}{ Poisson regression b (SE) } \\
\hline & & & & Unadjusted & Adjusted \\
\hline \multirow{14}{*}{$\begin{array}{l}\text { Knowledge index of smoking } \\
\text { harms including stroke, lung } \\
\text { cancer in smokers and } \\
\text { cardiovascular disease }\end{array}$} & Model 1 & & & & \\
\hline & Control cities & 1.37 & 1.63 & ref & ref \\
\hline & Intervention cities & 1.38 & 1.67 & $0.013(0.034)$ & $0.045(0.038)$ \\
\hline & Model 2 & & & & \\
\hline & Not recalled & 1.36 & 1.62 & ref & ref \\
\hline & Recalled the campaign & 1.51 & 1.97 & $0.168(0.043)^{* * *}$ & $0.147(0.043)^{* *}$ \\
\hline & Model 2 & & & & \\
\hline & Not recalled & 1.36 & 1.62 & ref & ref \\
\hline & Recalledt 1 channel & 1.51 & 1.89 & $0.124(0.046)^{*}$ & $0.093(0.043)^{*}$ \\
\hline & $\begin{array}{l}\text { Recalled } 2 \text { and more channels } \\
\text { Model } 4\end{array}$ & 1.45 & 2.24 & $0.301(0.069)^{* * *}$ & $0.259(0.066)^{* * *}$ \\
\hline & Not recalled $\ddagger$ & 1.36 & 1.62 & ref & ref \\
\hline & Recalled TV & 1.51 & 2.02 & $0.131(0.044)^{* *}$ & $0.106(0.040)^{*}$ \\
\hline & Recalled poster & 1.30 & 2.10 & $0.152(0.074)^{*}$ & $0.0109(0.082)$ \\
\hline & Recalled mobile media & 1.46 & 2.14 & $0.089(0.071)$ & $0.087(0.079)$ \\
\hline
\end{tabular}

Adjusted for age group, sex, income, education, marital status, smoking status, intention to quit, exposure to general anti-smoking campaign and survey time from the campaign end until the time of the post-campaign survey.

Statistically significant levels for ordinal regression: ${ }^{*} \mathrm{p}<0.05 ;{ }^{* *} \mathrm{p}<0.01 ;{ }^{* * *} \mathrm{p}<0.001$.

tParticipants ( $n=51$ ) who recalled the campaign but did not report any channel through which they saw the campaign were removed from the analysis.

¥This analysis is a single model in which dummy variables were created for each channel, with no recall of any exposure to the campaign as the reference group.

TV, television.

been exposed to campaign materials. Future campaign evaluations should assess recall with more optimal evaluation time frames and better control for contamination issues.

As expected, participants in the intervention cities who recalled the campaign via one or more channels had significantly greater levels of campaign-targeted knowledge of smoking harms. This finding supports the idea that multimedia interventions may have provided greater opportunities to learn and remember new health effects but the campaign effect may not have been large enough for the effects to influence attitudes. Only recall via a single channel was significantly associated with disapproval of cigarette gifts, with TV likely to be the primary source of these overall effects. These results should be interpreted with some caution given the small number of participants who recalled exposure through more than one channel. TV appears to have been a more effective medium than print and mobile media for reaching and influencing smokers, which is consistent with previous studies. ${ }^{2}{ }^{3}$ Nevertheless, statistically significant effects were limited to changes in knowledge. The lack of effects for print and mobile media may be also due to small sample sizes of those who recalled the campaign through these channels, thus we were unable to detect statistically significant differences.

Campaign-targeted attitudes towards cigarette gifts were assessed only after the campaign took place, and therefore reverse causality (ie, smokers who already disapproved of cigarette gifts tended to notice or recall the campaign ad, or participants in the intervention cities already disapproved cigarettes more than those in the control cities) may explain our findings. Future campaign evaluations should include preassessment and postassessment of attitudes to make valid causal conclusions.

Recall of the campaign was low (14\%), which may have been due to the relatively short duration of the campaign, low campaign intensity and lengthy time from the campaign end to the follow-up survey. The duration of the campaign, at least through electronic media, was only four weeks. Gross rating points and targeted rating points as a standard measure of campaign reach and frequency were unavailable to assess population exposure to address low self-reported recall rates. The postcampaign survey was conducted three months after the campaign broadcast ended, and lasted for five months, which may have resulted in the decay of campaign effects. ${ }^{2}$ Future campaign evaluations need to include the assessment of population exposure by gross rating points to understand campaign intensity.

In addition to those already mentioned, several limitations should be noted. The sample was designed to be representative of urban cities in China, therefore results should not be generalised to the rural Chinese population. The pre-evaluation and post-evaluation surveys were not conducted immediately before and after the GCGH campaign because this study used surveys from the ITC China Project, which was designed to measure the effectiveness of national-level tobacco control policies, rather than the campaign alone. ${ }^{29}$ The lengthy period between precampaign and post-campaign surveys may have introduced some biases. First, the pre-campaign survey was conducted 1 year before the campaign was broadcast, which is not optimal given that changes may have taken place between the pre-campaign survey and campaign onset. Second, the short duration of the campaign and the lengthy time from the campaign end to postcampaign survey (3-7 months) may have missed the maximal impact of the campaign as campaign effects decay. ${ }^{2}$ But our analyses adjusted for the length of surveying, the time between the campaign end and the post-campaign survey. Studies show that the beneficial effect of mass media campaigns appears only within 2-3 months after exposure. ${ }^{76}$ Indeed, this likely helps to explain the low campaign recall rate. Third, this study is subject to internal validity threats such as history effects due to the lengthy evaluation timeframe. For example, the SFO initiatives which included mass media campaigns promoting smokefree environments took place in Beijing, Shanghai and Shenyang before and during Olympics games in August 2008. The time frame of the SFB campaign (February 2008 to February 2009 in Beijing) also overlapped with the GCGH campaign. To address the possible influence of these events on study outcomes, our adjusted models included statistical controls for exposure to any anti-smoking campaigns in the last six months. Despite these problems of timing and length, the biases are really conservative since we were able to detect campaign effects.

The potential non-comparability of the intervention cities and control cities such as tobacco industry activities and economic 
development may also have confounded associations between study variables. For example, Changsha is a mid-sized, major cigarette-producing city and Yinchuan is a small, economically less developed city while the four intervention cities are among the top 10 largest cities in China. Although our regression analyses controlled for measured differences between comparison groups, unmeasured variables may also explain the results. Furthermore, contamination is a potential internal validity threat since participants in the control cities could have been exposed to the campaign messages outside the city where they live or through satellite TV within their city of residence. Our assessment of campaign exposure within intervention cities helps overcome this limitation, but nevertheless may be limited by recall bias. Future campaign evaluations should better monitor and address contamination issues and control for non-comparability of comparison groups. More optimal evaluation timeframes could better assess recall, and overcome issues regarding small sample sizes for levels and types of exposure. In spite of these issues, our study is suggestive of campaign effects. Future research should include measurement of key behavioural outcomes. For example, the GCGH campaign's primary message involved cigarette gifting behaviour, and questions should have assessed the prevalence and incidence of giving and receiving cigarettes as gifts.

\section{CONCLUSIONS}

This study strengthens the evidence that mass media campaigns with graphic, emotionally evocative messages can raise awareness of smoking harms and change smokers' attitudes that are favourable to smoking-related norms. Our study suggests that the Chinese government should consider regularly rerunning the GCGH campaign at greater intensity and for longer duration, and develop similar campaigns to address the tobacco epidemic in China. Those campaigns should be accompanied by rigorous evaluations to better evaluate the messages and media channels that are most effective at reaching and influencing people to adopt healthy behaviour.

\section{What this paper adds}

- The enormity of the tobacco epidemic in China calls for multiple approaches to increasing knowledge of the harms of cigarettes and changing societal norms about cigarettes. Mass media campaigns are one possible strategy, and such approaches are just beginning in China.

- This paper reports the results of the first large-scale populationbased evaluation of a tobacco control mass media campaign conducted in China. The mass media campaign targeted the cultural tradition of gifting cigarettes at the same time as it aimed to increase knowledge about smoking-related harms.

- The longitudinal evaluation conducted among a population-based representative sample demonstrates that as in many other countries, mass media campaigns in China can be effective in raising awareness of the harms of cigarettes.

- Such campaigns can also be effective in denormalising common cultural practices that serve to maintain positive norms around tobacco products, such as giving cigarettes as gifts in China.

Acknowledgements The authors thank the Chinese Center for Disease Control and Prevention (CDC), the local CDC staff, other groups and individuals who assisted in the conduct of the study. The authors also thank the World Lung Foundation for providing information about the campaign development materials and media plans for the GCGH campaign.
Contributors L-LH devised the study, conducted the analyses and oversaw the writing of the manuscript. JFT helped develop the analysis plan and helped write the manuscript. GTF, QL, YJ, YC, KMW and DBF contributed to the manuscript by providing key information on the study context, suggestions about the analysis approach and interpretation of results. All authors read, provided comments and approved the final manuscript.

Funding The ITC China Project was supported by grants from the US National Cancer Institute (R01 CA125116 and P01 CA138389), the Roswell Park Transdisciplinary Tobacco Use Research Center (P50 CA111236), the Robert Wood Johnson Foundation (045734); the Canadian Institutes of Health Research (57897, 79551 and 115016), and the Chinese Center for Disease Control and Prevention. Additional support was provided to Geoffrey T Fong from a Senior Investigator Award from the Ontario Institute for Cancer Research and a Prevention Scientist Award from the Canadian Cancer Society Research Institute. The funding sources had no role in the study design, in collection, analysis and interpretation of data, in the writing of this paper, or in the decision to submit the paper for publication.

Competing interest None declared.

Ethics approval Ethics approval was obtained from the Office of Research at the University of Waterloo (Waterloo, Canada) and the Institutional Review Boards at the Roswell Park Cancer Institute (Buffalo, USA), the Cancer Council Victoria (Victoria, Australia), and the Chinese Center for Disease Control and Prevention (Beijing, China).

Provenance and peer review Not commissioned; externally peer reviewed.

\section{REFERENCES}

1 World Health Organization. WHO Report on the Global Tobacco Epidemic, 2011 -Warning about the dangers of tobacco. Geneva: World Health Organization, 2011. http://www.who.int/tobacco/global_report/2011/en/ (accessed 1 May 2013).

2 Durkin SJ, Brennan E, Wakefield MA. Mass media campaigns to promote smoking cessation among adults: an integrative review. Tob Control 2012;21:127-38.

3 National Cancer Institute. The role of media in promoting and reducing tobacco use. Tobacco control monograph No. 19. Bethesda, MD: US Department of Health and Human Services, National Institute of Health, National Cancer Institute, 2008.

4 Siegel M. Antismoking advertising: figuring out what works. J Health Communication 2002;7:157-62.

5 Farrelly MC, Davis KC, Haviland L, et al. Evidence of a dose-response relationship between "truth" anti-smoking ads and youth smoking prevalence. Am J Public Health 2005:95:425-31.

6 Goldman LK, Glantz SA. Evaluation of anti-smoking advertising campaigns. JAMA 1998;279:772-7.

7 Wakefield MA, Durkin SJ, Spittal MJ, et al. Impact of tobacco control policies and mass media campaigns on monthly adult smoking prevalence. Am J Public Health 2008;98:1443-50.

8 Thrasher JF, Huang L, Perez-Hmandez R, et al. Evaluation of a social marketing campaign to support Mexico City's comprehensive smoke-free law. Am J Public Health 2011;101:328-35.

9 Wakefield MA, Bayly M, Durkin SJ, et al. Smokers' responses to television advertisements about the serious harms of tobacco use pre-testing results from 10 low- to middle-income countries. Tob Control 2013;22:24-31.

10 Durkin SJ, Biener L, Wakefield MA. Effects of different types of anti-smoking ads on reducing disparities in smoking cessation among socioeconomic subgroups. Am J Public Health 2009;99:2217-23.

11 Durkin SJ, Wakefield MA, Spittal JS. Which types of televised anti-tobacco campaigns prompt more quitline calls from disadvantaged groups? Health Educ Res 2011;26:998-1009.

12 Davis KC, Nonnemaker JM, Farrelly MC, et al. Exploring differences in smokers' perceptions of the effectiveness of cessation media messages. Tob Control 2011;20:26-33.

13 Murukutla N, Turk T, Prasad CV, et al. Results of a national mass media campaign in India to warn against the dangers of smokeless tobacco consumption. Tob Control 2011;21:12-7.

14 Perl R, Stebenkova L, Morozova I, et al. Mass media campaign within reach: effective efforts with limited resources in Russia's capital city. Tob Control 2011;20:439-41.

15 Chinese Center for Disease Control and Prevention. Global Adult Tobacco Survey (GATS) China 2010 Country Report. Beijing, China: Chinese Center for Disease Control and Prevention, 2010. http://www.who.int/tobacco/surveillance/survey/gats/ en_gats_china_report.pdf?ua=1 (accessed $30 \mathrm{Apr} 2014$ ).

16 Eriksen $\bar{M}$, Mackay J, Ross H. The tobacco atlas. 4th edn. Atlanta: American Cancer Society, 2012

17 Yang G. China wrestles with tobacco control: interview by Weiyuan Cui. Bull World Health Organ 2010;88:251-52.

18 Loo D. China's tobacco monopoly bigger by profit than HSBC. Bloomberg News 2012. http://www.bloomberg.com/news/2012-03-06/china-s-tobacco-monopolybigger-by-profit-than-hsbc.html (accessed 1 May 2013). 


\section{Research}

19 Hu TW, Mao Z, Ong M, et al. China at the crossroads: the economics of tobacco and health. Tob Control 2006;15:i37-41.

20 Hu TW. Tobacco control policy analysis in China: economics and health. Singapore: World Scientific Publishing Co, 2008.

21 Li C. The political mapping of China's tobacco industry and anti-smoking campaign. Washington, D.C.: The Brookings Institution, 2012.

22 Shi Y, Zhao BF, Liu Y, et al. The impact of the "Sponge" advertisement among adult residents in Kunming City. (In Chinese). Soft Sci Health 2010: 24;332-4.

23 Wang $\mathrm{C}$. Evaluation of the long-term effects of the Smoke Free Olympics Initiatives in Beijing, Shanghai, and Shenyang: findings from the ITC China Study. The 15th World Conference on Tobacco or Health, Singapore, March 20-24, 2012.

24 ITC Project and Office of Tobacco Control, China CDC. ITC China Project Report. Findings from the Wave 1 to 3 Surveys (2006-2009). University of Waterloo, Waterloo, Ontario, Canada, and Office of Tobacco Control, Chinese Center for Disease Control and Prevention, Beijing, China. Beijing: China Modern Economic Publishing House 2012

25 Chu A, Jiang N, Glantz SA. Transnational tobacco industry promotion of the cigarette gifting custom in China. Tob Control 2011;20:e3.

26 Huang L, Thrasher JF, Jiang Y, et al. Incidence and correlates of receiving cigarettes as gifts and selecting preferred brand because it was gifted: Findings from the ITC China Survey. BMC Public Health 2012;12:996.

27 Hu M, Rich ZC, Luo D, et al. Cigarette sharing and gifting in rural China: a focus group study. Nicotine Tob Res 2012;14:361-7.
28 Alday J. Survey indicates fewer people in Beijing and Shanghai intended to give cigarettes as gifts after seeing mass media campaign. World Lung Found 2009. http:// www.worldlungfoundation.org/ht/d/ReleaseDetails/i/6253 (accessed 1 May 2013).

29 Wu CB, Thompson ME, Fong GT, et al. Methods of the International Tobacco Control (ITC) China Survey. Tob Control 2010;19:11-5.

30 ITC Southeast Asia China Research Team, ITC Southeast Asia International Planning Group. International Tobacco Control China Survey: Wave 1 (2006) ITC China Technical Report. Waterloo: University of Waterloo, 2008.

31 World Lung Foundation, Chinese Center for Disease Control and Prevention, \& World Health Organization. Giving cigarettes=giving harm. [Video]. 2009. http://67. 199.72.89/mmr/english/ad_giftGiving.html. (accessed 25 Nov 2013).

32 World Lung Foundation, Chinese Center for Disease Control and Prevention, \& World Health Organization. Giving cigarettes is giving harm. [Journal Cover]. 2009. http:/l tobaccocontrol.bmj.com/content/19/1.cover-expansion. (accessed 25 Nov 2013).

33 Mullin S, Prasad V, Kaur J, et al. Increasing evidence for the efficacy of tobacco control mass media communication programming in low- and middle-income countries. J Health Commun 2011;16:49-58.

34 Heatherton TF, Kozlowski LT, Frecker RC, et al. Measuring the heaviness of smoking: using self-reported time to the first cigarette of the day and number of cigarettes smoked per day. Addiction 1989;84:791-800.

35 StataCorp LP. Stata/IC 11.2 for Windows. College Station, TX: StataCorp LP, 2009.

36 Wakefield MA, Spittal MJ, Yong $\mathrm{H}$, et al. Effects of mass media campaign exposure intensity and durability on quit attempts in a population-based cohort study. Health Educ Res 2011;26:988-97. 